\title{
Health Diagnosis Expert Advisory System on Trained Data Sets for Hyperthyroid
}

\author{
V Prasad \\ Associate Professor \\ Department of CSE \\ Raghu Institute of Technology
}

\author{
T Srinivasa Rao \\ Associate Professor \\ Department of CSE \\ GITAM Institute of Technology
}

\author{
A Veera Reddy,B Chaitanya \\ Final B.Tech \\ Department of CSE \\ Raghu Institute of Technology
}

\begin{abstract}
This paper presents a collection of 28 pristine symptoms which are used for the identification of Hyperthyroid disease which are heartwarming to humankind. Ghastly, Hyperthyroid affect people without being noticed until the end. In this Health Diagnose Expert Advisory System (HDEAS) we proposed a method for diagnosing the Hyperthyroid disease by enabling a list of symptoms that the person is likely to suffer from. Here the diagnosis is done by the method of prediction using Trained Data Sets(TDS) and the results are compared by using suitable Data Matching Systems (DMS).The TDS are provided by Intelligent System Laboratory of K.N.Toosi University of Technology, Imam Khomeini Hospital .Proceedings of this research showed that HDEAS can be used effectively. The acquainted knowledge is represented in the diagrams, charts and tables. The database consists of four wide classifications of Thyroid Disease, with well-organized pattern structure of symptoms. By providing an affable interface, user can input the data in the questionnaire form developed. This work predicts the actual levels of the hyperthyroid in human body.
\end{abstract}

\section{General Terms}

Hyperthyroid Disease, Expert Advisory System ,K N Toosi University Datasets, Machine Learning Algorithms.

\section{Keywords}

Data Matching System, Health Expert Advisory System, Knowledge Base, Prediction, Trained Data Sets, UCI Machine Learning Data Sets.

\section{INTRODUCTION}

Hyperthyroid [1] is one of the most widespread diseases that are frequently misunderstood and misdiagnosed. Hyperthyroid develops when the body is exposed to excessive amounts of Thyroid hormone. This disorder occurs in almost one percent of all Americans and affects women, five to ten times more often than men. In its mildest form, Hyperthyroid may not cause identifiable symptoms. More often, however, the symptoms are thwarting, disabling or even critical. Due to the advent of developing technology and information in medical sciences, the computer science professionals are capable of providing Expert Advisory System (EAS)[1][3] to diagnose different kinds of diseases with high accuracy. These systems are based on Artificial Intelligence techniques which help the doctors to minimize the cost and time in effective diagnose. Inspired with the above said, we proposed a method for diagnosing disease using TDS.[4] The users can use this EAS to know the disease which they are suffering by entering the symptoms. The doctors can use the TDS after essential clinical authorization for better diagnosis. Therefore all the stake holders connected with Health Science will be benefited with this web portal. The main components of an Expert System are: Knowledge base [12] and Inference Engine.

\subsection{Knowledge base about Domain}

This system will enhance the Human Life by bridging the gap where there is lack of expert doctors, it also provide immediate outputs with the best optimistic values obtained from Tested Data Sets (TDS) provided by Intelligent System Laboratory of K.N.Toosi University of Technology from Imam Khomeini Hospital.[4] Inference engine - draws conclusions from the knowledge base.

\subsection{Elements of our Expert System are}

\subsubsection{User interface}

It includes 28 symptoms leading to various thyroid related diseases they are Hyperthyroid, Hypothyroid, Goiter, T3 toxic and the various tests that are performed to diagnose. [1]

\subsubsection{Working memory}

Data base consisting of Trained Datasets.

\subsubsection{Inference engine}

Inferences a conclusion using Prediction[1-3] by making a proportional analysis of the TDS.[3]

TDS are lists of variables composed to meet up the minimal requirements of the group's goals, often with an additional list of elements that are recommended for the most effective operation. Required data sets are not the same for all standard setters.

\section{PROPOSED SYSTEM}

Health is a resource for everyday life, not the intention of living and "Health is a positive perception emphasizing social and personal resources, as well as physical capacities". The proposed system aims to provide an imprecise diagnosis of the four thyroid related diseases such as Hyperthyroid in particular, Hypothyroid, goiter and T3 Toxic by accepting the values fed by the user, from the user interface where all the possible symptoms of thyroid are segregated, assessed and a final list of 28 symptoms is provided to the user. Once the user enters the details of the symptoms and the tests that he had undergone such as TSH, T3, TT4, T4U, FTI, TBG the values are compared against the TDS and a possible assumption is made.

The System developed is used for Offline Web \& Analysis on Hyperthyroid [3] and related diseases. The system is developed by using DMS [13] which is applied on TDS to identify the relevant disease according to the data of the symptoms specified in the knowledge base. Once the user enters the details of the symptoms and submits, then it 
predicts the Human disease which the user is suffering from and gives the referral source as the nearest hospital for further proceedings. Since the entire work is based on the TDS utmost care is taken while acquiring the information regarding the disease and a collection of about 2800 sets are coined. Since the acquired information is huge the chance of insufficiency of data in the knowledge base is of lesser concern. The functionality of this HDEAS is merely based on an optimistic approach towards the prediction of the disease. This work can predict a disease with an efficiency of about $65 \%$. However, this needs to be further enhanced using suitable Machine Learning and Advanced Artificial Intelligence Optimization Algorithms, [5] [7] [14] as prediction mechanism with an insufficient database may not be a considerable constraint in optimized approach but for a complete clinically authorized Expert System [5] every constraint is of great importance. The DMS produces a considerably acceptable prediction when there are prominent hits for the knowledge base. Since the TDS are statistically huge the resultant successful predictions are considered to be effective and efficient.

\section{SYSTEM DESIGN}

In the development of this Offline web portal we had implemented the below said

a) Data Matching System Using TDS [13].

b) Event Flow Diagram

\subsection{Event Flow Diagram}

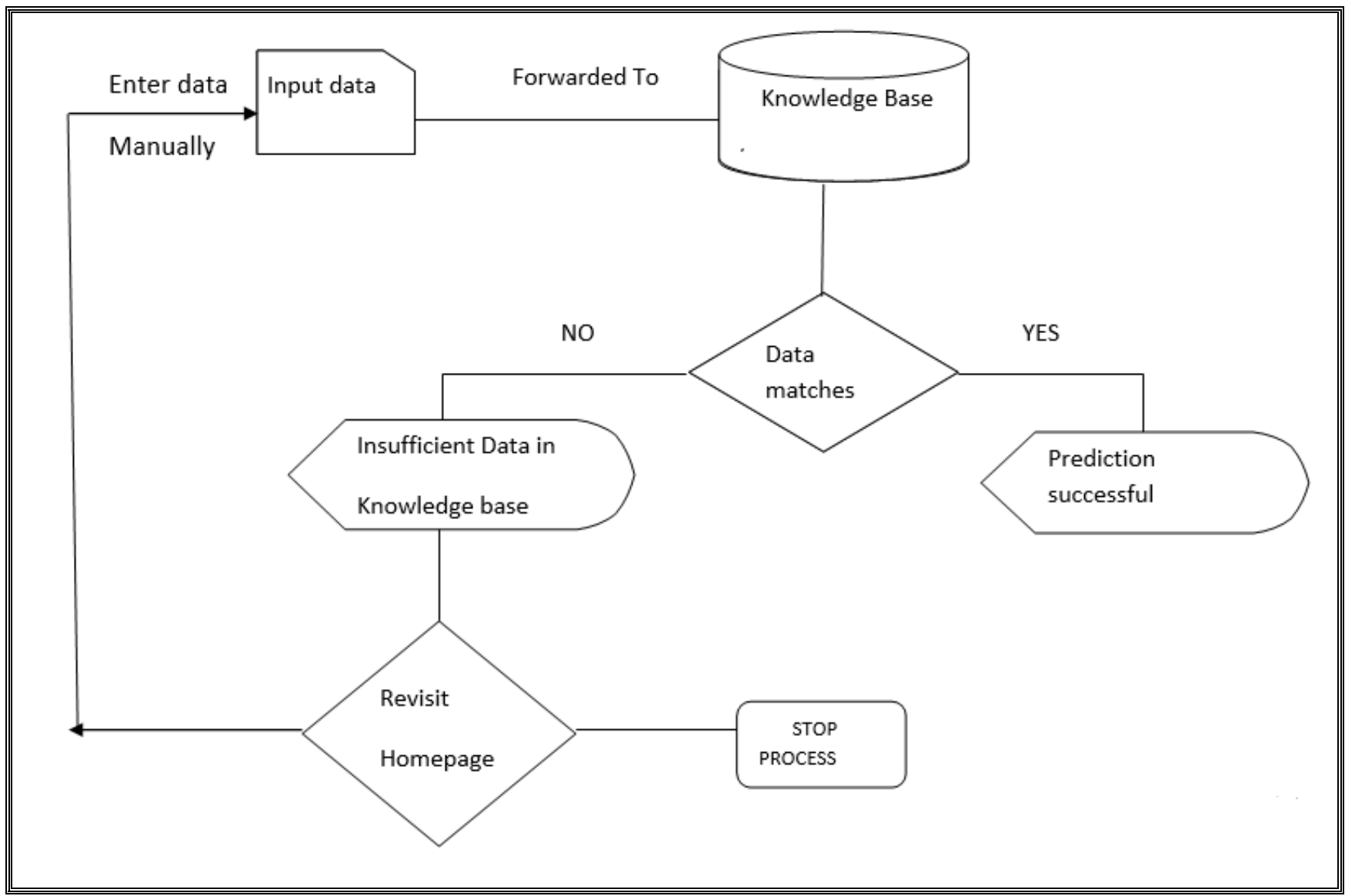

Fig 1: Event Flow/ User Interface Diagram

Figure 1: The above diagram gives us the User Interface diagram representation which consists of the flow events. In this event flow diagram, we can identify the Data Matching System with controlled data of Rule Based System [1].

\subsection{Functional Requirements}

The following are the functional requirements of this system:

Inputs: The system needs the information about

- About Thyroid Diseases

- $\quad$ Thyroid Disease Classification[5][6][15]

- Common Thyroid Disease Symptoms[4]

Outputs::The outputs of the system will be:

- Diagnosis of the disease

- $\quad$ Referral source[4]
Store the information collected through inputs is stored as a knowledge base that serves as a repository[4] for quick processing and future retrieval.

Computations various computations are to be performed while evaluating and generating reports based on the requirement and information collected. These are nothing but forward chaining and backward chaining etc.

\subsection{Non Functional Requirements}

Platform is equipped with Java Server Pages and Net Beans. Technology used is JAVA (JSP Front End) \& MYSQL (Rear End) 
This is a web enabled application developed using JSP and Datasets. So as to ensure the quality of the software, all software engineering [9] concepts, including test cases are implemented. This depicts the client server architecture and forms a well set layout.

\subsection{Knowledge Base}

Knowledge Base [11] is obtained from the Intelligent System Laboratory of K.N.Toosi University of Technology from Imam Khomeini hospital the following are the attributes taken into consideration for the TDS:

$\begin{array}{ll}\text { S01:: Age } & \text { S02:: Sex } \\ \text { S03:: On Thyroxin } & \text { S04:: Query On Thyroxin } \\ \text { S05:: On Anti-Thyroid Medication } & \\ \text { S06:: Sick } & \text { S07:: Pregnant } \\ \text { S08:: Thyroid Surgery } & \text { S09:: I131 Treatment } \\ \text { S10:: Query Hypothyroid } & \text { S11:: Query Hyperthyroid } \\ \text { S12:: Lithium } & \text { S13:: Goiter } \\ \text { S14:: Tumor } & \text { S15:: Hypo Pituitary } \\ \text { S16:: Psych } & \text { S17:: TSH Measured } \\ \text { S18:: TSH Value } & \text { S19:: T3 Measured } \\ \text { S20:: T3 Value } & \text { S21:: TT4 Measured } \\ \text { S22:: TT4 Value } & \text { S23:: T4U Measured } \\ \text { S24:: T4U Value } & \text { S25:: FTI Measured } \\ \text { S26:: FTI Value } & \text { S27:: TBG Measured } \\ \text { S28:: TBG } & \text { S29:: Referral Source }\end{array}$

\section{RESULTANT ANALYSIS}

Rule 1: IF

$\begin{array}{llllll}\text { S01 }=41 & \text { S02 }=F & \text { S03 }=F & \text { S04 }=F & \text { S05 }=F & \text { S06 }=F \\ \text { S07=F } & \text { S08=F } & \text { S09=F } & \text { S10=F } & \text { S11=F } & \text { S12=F } \\ \text { S13=F } & \text { S14=F } & \text { S15=F } & \text { S16=F } & \text { S17=T } & \text { S18=1.3 } \\ \text { S19=T } & \text { S20=2.5 } & \text { S21=T } & \text { S22=125 } & \text { S23=T } & \text { S24=1.14 } \\ \text { S25=T } & \text { S26=109 } & \text { S27=F } & \text { S28=? } & & \end{array}$

Disease Predicted=Negative.|3733

Referral Source is $=\mathrm{SVHC}$

Rule 2: IF

$\begin{array}{llllll}\text { S01 }=87 & \text { S02 }=F & \text { S03 }=F & \text { S04 }=F & \text { S05 }=F & \text { S06 }=F \\ \text { S07=F } & \text { S08=F } & \text { S09=F } & \text { S10=F } & \text { S11=F } & \text { S12=F } \\ \text { S13=F } & \text { S14=F } & \text { S15=F } & \text { S16=F } & \text { S17=T } & \text { S18=0.15 } \\ \text { S19=T } & \text { S20=1.7 } & \text { S21=T } & \text { S22=162 } & \text { S23=T } & \text { S24=0.87 } \\ \text { S25=T } & \text { S26=186 } & \text { S27=F } & \text { S28=? } & & \end{array}$

Disease Predicted=Hyperthyroid.|1873

Referral Source is $=$ SVI

Rule 3: IF

$\begin{array}{llllll}\text { S01 }=44 & \text { S02 }=\text { M } & \text { S03=F } & \text { S04=F } & \text { S05=F } & \text { S06=F } \\ \text { S07=F } & \text { S08=F } & \text { S09=F } & \text { S10=F } & \text { S11=F } & \text { S12=F } \\ \text { S13=F } & \text { S14=F } & \text { S15=F } & \text { S16=F } & \text { S17=T } & \text { S18=2 } \\ \text { S19=T } & \text { S20=1.3 } & \text { S21=T } & \text { S22=136 } & \text { S23=T } & \text { S24=0.94 } \\ \text { S25=T } & \text { S26=145 } & \text { S27=F } & \text { S28=? } & & \end{array}$

Disease Predicted=Negative. $\mid 3345$

Referral Source is $=$ SVHD

Rule 4: IF

$\begin{array}{llllll}\text { S01=19 } & \text { S02=F } & \text { S03=F } & \text { S04=F } & \text { S05=F } & \text { S06=F } \\ \text { S07=T } & \text { S08=F } & \text { S09=F } & \text { S10=F } & \text { S11=F } & \text { S12=F } \\ \text { S13=F } & \text { S14=T } & \text { S15=F } & \text { S16=F } & \text { S17=T } & \text { S18=0.45 }\end{array}$

$\mathrm{S} 19=\mathrm{T} \quad \mathrm{S} 20=3.2 \quad \mathrm{~S} 21=\mathrm{T} \quad \mathrm{S} 22=130 \mathrm{~S} 23=\mathrm{T} \quad \mathrm{S} 24=1.83$ $\mathrm{S} 25=\mathrm{T} \quad \mathrm{S} 26=71 \quad \mathrm{~S} 27=\mathrm{F} \quad \mathrm{S} 28=$ ?

Disease Predicted $=$ Goitre. $\mid 3523$

Referral Source is $=$ STMW

Rule 5: IF

S01 $=25 \quad$ S02 $=F \quad$ S03 $=F \quad$ S04 $=F \quad$ S05 $=F \quad$ S06 $=F$ S07 $=F \quad S 08=F \quad S 09=F \quad S 10=F \quad S 11=F \quad S 12=F$ $\mathrm{S} 13=\mathrm{F} \quad \mathrm{S} 14=\mathrm{F} \quad \mathrm{S} 15=\mathrm{F} \quad \mathrm{S} 16=\mathrm{F} \quad \mathrm{S} 17=\mathrm{T} \quad \mathrm{S} 18=1.6$ $\mathrm{S} 19=\mathrm{T} \quad \mathrm{S} 20=5.4 \quad \mathrm{~S} 21=\mathrm{T} \quad \mathrm{S} 22=152 \quad \mathrm{~S} 23=\mathrm{T} \quad \mathrm{S} 24=1.5$ $\mathrm{S} 25=\mathrm{T} \quad \mathrm{S} 26=102 \mathrm{~S} 27=\mathrm{F} \quad \mathrm{S} 28=$ ?

Disease Predicted=Negative.|1183

Referral Source is $=\mathrm{STMW}$

Rule 6: IF

S01 $=60 \quad$ S02 $=M \quad S 03=F \quad S 04=F \quad$ S05 $=T \quad$ S06 $=F$

S07 $=F \quad$ S08 $=F \quad$ S09 $=F \quad$ S10 $=F \quad$ S11 $=F \quad$ S12 $=F$

$\mathrm{S} 13=\mathrm{F} \quad \mathrm{S} 14=\mathrm{F} \quad \mathrm{S} 15=\mathrm{F} \quad \mathrm{S} 16=\mathrm{F} \quad \mathrm{S} 17=\mathrm{T} \quad \mathrm{S} 18=0.2$

$\mathrm{S} 19=\mathrm{T} \quad \mathrm{S} 20=4 \quad \mathrm{~S} 21=\mathrm{T} \quad \mathrm{S} 22=68 \quad \mathrm{~S} 23=\mathrm{T} \quad \mathrm{S} 24=1$

S25 $=\mathrm{T} \quad \mathrm{S} 26=67 \quad \mathrm{~S} 27=\mathrm{F} \quad \mathrm{S} 28=$ ?

Disease Predicted $=$ T3 Toxic. $\mid 547$

Referral Source is $=$ other.

Rule 7: IF

S01 $=29 \quad$ S02 $=F \quad$ S03 $=F \quad$ S04 $=F \quad$ S05 $=F \quad$ S06 $=F$ S07 $=\mathrm{T} \quad$ S08 $=\mathrm{F} \quad$ S09 $=\mathrm{F} \quad \mathrm{S} 10=\mathrm{F} \quad \mathrm{S} 11=\mathrm{F} \quad \mathrm{S} 12=\mathrm{F}$ $\mathrm{S} 13=\mathrm{F} \quad \mathrm{S} 14=\mathrm{T} \quad \mathrm{S} 15=\mathrm{F} \quad \mathrm{S} 16=\mathrm{F} \quad \mathrm{S} 17=\mathrm{T} \quad \mathrm{S} 18=1.4$ $\mathrm{S} 19=\mathrm{T} \quad \mathrm{S} 20=3.4 \quad \mathrm{~S} 21=\mathrm{T} \quad \mathrm{S} 22=147 \mathrm{~S} 23=\mathrm{T} \quad \mathrm{S} 24=1.49$ S25 $=\mathrm{T} \quad$ S26 $=99 \quad$ S27 $=F \quad$ S 28=?

Disease Predicted=goiTre. $\mid 2469$

Referral Source is

Rule 8: IF

$\begin{array}{llllll}\text { S01=53 } & \text { S02=M } & \text { S03=F } & \text { S04=F } & \text { S05=F } & \text { S06=F } \\ \text { S07=F } & \text { S08=F } & \text { S09=F } & \text { S10=F } & \text { S11=F } & \text { S12=F } \\ \text { S13=F } & \text { S14=F } & \text { S15=F } & \text { S16=F } & \text { S17=T } & \text { S18=1.4 } \\ \text { S19=T } & \text { S20=1.9 } & \text { S21=T } & \text { S22=104 } & \text { S23=T } & \text { S24=0.93 } \\ \text { S25=T } & \text { S26=112 } & \text { S27=F } & \text { S28=? } & & \end{array}$

Disease Predicted=Negative. $\mid 43$

Referral Source is=SVI

Rule 9: IF

S01 $=41 \quad S 02=F \quad S 03=F \quad$ S04 $=F \quad$ S05 $=F \quad$ S06 $=F$ $\mathrm{S} 07=\mathrm{F} \quad \mathrm{S} 08=\mathrm{F} \quad \mathrm{S} 09=\mathrm{F} \quad \mathrm{S} 10=\mathrm{F} \quad \mathrm{S} 11=\mathrm{T} \quad \mathrm{S} 12=\mathrm{F}$ $\mathrm{S} 13=\mathrm{F} \quad \mathrm{S} 14=\mathrm{F} \quad \mathrm{S} 15=\mathrm{F} \quad \mathrm{S} 16=\mathrm{F} \quad \mathrm{S} 17=\mathrm{T} \quad \mathrm{S} 18=0.2$ $\mathrm{S} 19=\mathrm{T} \quad \mathrm{S} 20=3.8 \quad \mathrm{~S} 21=\mathrm{T} \quad \mathrm{S} 22=253 \quad \mathrm{~S} 23=\mathrm{T} \quad \mathrm{S} 24=1.24$ $\mathrm{S} 25=\mathrm{T} \quad \mathrm{S} 26=204 \mathrm{~S} 27=\mathrm{F} \quad \mathrm{S} 28=$ ?

Disease Predicted=Hyperthyroid.|2003 Referral Source is=STMW.

Rule 10: IF

S01 $=79 \quad$ S02 $=M \quad$ S03 $=F \quad$ S04 $=F \quad$ S05 $=F \quad$ S06 $=F$ S07 $=F \quad$ S08 $=F \quad$ S09 $=F \quad$ S10 $=F \quad$ S11 $=F \quad$ S12 $=F$ S13 $=F \quad S 14=F \quad S 15=F \quad S 16=F \quad S 17=T \quad S 18=0.03$ $\mathrm{S} 19=\mathrm{T} \quad \mathrm{S} 20=4.1 \quad \mathrm{~S} 21=\mathrm{T} \quad \mathrm{S} 22=160 \mathrm{~S} 23=\mathrm{T} \quad \mathrm{S} 24=0.78$ S25 $=\mathrm{T} \quad$ S26 $=204$ S27 $=F \quad$ S28 $=$ ?

Disease Predicted=Hyperthyroid.|1086

Referral Source is $=$ other. 


\section{DATASET}

The Datasets mainly consisting of 28 Symptoms with which we can predict the existence of disease levels and predict them.

Table 1. Table consists of Dataset Sample used for Prediction of Hyperthyroid

\begin{tabular}{|c|c|c|c|c|c|c|c|c|c|c|c|c|c|c|c|c|}
\hline SYMPTOMS & S1 & S2 & S3 & S4 & S5 & S6 & S7 & S8 & S9 & S10 & S11 & S12 & S13 & S14 & S15 & S16 \\
\hline Rule SET-1 & 41 & F & F & F & F & F & F & F & F & F & F & F & F & F & F & F \\
\hline Rule SET-2 & 87 & F & F & F & F & F & F & F & F & F & F & F & F & F & F & F \\
\hline Rule SET-3 & 44 & M & F & F & F & T & F & F & F & F & F & F & F & F & F & F \\
\hline Rule SET-4 & 19 & F & F & F & F & F & T & F & F & F & F & F & F & T & F & F \\
\hline Rule SET-5 & 25 & F & F & F & F & F & F & F & F & F & F & F & F & F & F & F \\
\hline Rule SET-6 & 60 & M & F & F & T & F & F & F & F & F & F & F & F & F & F & F \\
\hline Rule SET-7 & 29 & F & F & F & F & F & T & F & F & F & F & F & F & T & F & F \\
\hline Rule SET-8 & 53 & M & F & F & F & F & F & F & F & F & F & F & F & F & F & F \\
\hline
\end{tabular}

\begin{tabular}{|c|c|c|c|c|c|c|c|c|c|c|c|c|c|}
\hline S17 & S18 & S19 & S20 & S21 & S22 & S23 & S24 & S25 & S26 & S27 & S28 & $\begin{array}{c}\text { Referral Source } \\
\text { (S29) }\end{array}$ & Predicted Disease (S30) \\
\hline $\mathrm{T}$ & 1.3 & $\mathrm{~T}$ & 2.5 & $\mathrm{~T}$ & 125 & $\mathrm{~T}$ & 1.14 & $\mathrm{~T}$ & 109 & $\mathrm{~F}$ & $?$ & SVHC & NEGATIVE.|3733 \\
\hline $\mathrm{T}$ & 0.15 & $\mathrm{~T}$ & 1.7 & $\mathrm{~T}$ & 162 & $\mathrm{~T}$ & 0.87 & $\mathrm{~T}$ & 186 & $\mathrm{~F}$ & $?$ & SVI & HYPERTHYROID.|1873 \\
\hline $\mathrm{T}$ & 2 & $\mathrm{~T}$ & 1.3 & $\mathrm{~T}$ & 136 & $\mathrm{~T}$ & 0.94 & $\mathrm{~T}$ & 145 & $\mathrm{~F}$ & $?$ & SVHD & NEGATIVE.|3345 \\
\hline $\mathrm{T}$ & 0.45 & $\mathrm{~T}$ & 3.2 & $\mathrm{~T}$ & 130 & $\mathrm{~T}$ & 1.83 & $\mathrm{~T}$ & 71 & $\mathrm{~F}$ & $?$ & STMW & GOITRE.|3523 \\
\hline $\mathrm{T}$ & 1.6 & $\mathrm{~T}$ & 5.4 & $\mathrm{~T}$ & 152 & $\mathrm{~T}$ & 1.5 & $\mathrm{~T}$ & 102 & $\mathrm{~F}$ & $?$ & STMW & NEGATIVE.|1183 \\
\hline $\mathrm{T}$ & 0.2 & $\mathrm{~T}$ & 4 & $\mathrm{~T}$ & 68 & $\mathrm{~T}$ & 1 & $\mathrm{~T}$ & 67 & $\mathrm{~F}$ & $?$ & OTHER & T3 TOXIC.|547 \\
\hline $\mathrm{T}$ & 1.4 & $\mathrm{~T}$ & 3.4 & $\mathrm{~T}$ & 147 & $\mathrm{~T}$ & 1.49 & $\mathrm{~T}$ & 99 & $\mathrm{~F}$ & $?$ & STMW & GOITRE.|2469 \\
\hline $\mathrm{T}$ & 1.4 & $\mathrm{~T}$ & 1.9 & $\mathrm{~T}$ & 104 & $\mathrm{~T}$ & 0.93 & $\mathrm{~T}$ & 112 & $\mathrm{~F}$ & $?$ & SVI & NEGATIVE.|43 \\
\hline
\end{tabular}

Table 1: (Description) After deciding the existence of symptoms, the disease is predicted and from that, the administrator would
Data Sets are purely obtained from Intelligent System Laboratory of K.N.Toosi University of Technology, Imam Khomeini Hospital.

\footnotetext{
decide and display the referral source, which is nearest Health Organization.
} 
6. RESULTS

\subsection{Case -1:}

\begin{tabular}{|c|c|c|}
\hline \multicolumn{3}{|c|}{ SELECT THE SYMPTOMS } \\
\hline Enter your age : & 87 & $\mathbf{v}$ \\
\hline Enter your Gender : & Male & - Female \\
\hline On Thyroxine: & 0 Yes & - No \\
\hline Query On Thyroxine: & Y Yes & (- No \\
\hline On Anti-Thyroid Medication : & Yes & - No \\
\hline Sick: & 0 Yes & (- No \\
\hline Thyroid Surgery : & Yes & (-) No \\
\hline I131 Treatment: & OYes & (- No \\
\hline Query Hypothyroid : & Yes & - No \\
\hline Query Hyperthyroid : & $\bigcirc$ Yes & (-) No \\
\hline Lithium: & Yes & (- No \\
\hline Goitre : & (1) Yes & (- No \\
\hline Tumor: & Yes & (-) No \\
\hline Hypopituitary : & Yes & ( ) No \\
\hline Psych: & Yes & (- No \\
\hline TSH Measured: & - Yes & No \\
\hline TSH : & 0.15 & \\
\hline T3 Measured: & - Yes & No \\
\hline T3: & 1.7 & \\
\hline T14 Measured: & - Y Yes & No \\
\hline TT4: & $\sqrt{162}$ & \\
\hline T4U Measured: & - Yes & No \\
\hline T4U: & 0.87 & \\
\hline FII Measured: & - Yes & No \\
\hline FTI : & 186 & \\
\hline TBG Measured: & 0 Yes & (-) No \\
\hline & & \\
\hline
\end{tabular}

Fig 2: Input Screen to Identify Hyperthyroid Disease

\section{Output:}

Report Generated as: Hyperthyroid.| 1873

Referral Source Specified: SVI

Figure 2: This screen shot shows the diagnosis of Hyperthyroid according to the symptoms selected by the user, the prediction is made as per the suggested methd and the refferal source SVI is suggested to the user for further proceedings of treatment.
6.2 Case-2:

\begin{tabular}{|c|c|c|}
\hline \multicolumn{3}{|c|}{ SELECT THE SYMPTOMS } \\
\hline Enter your age : & 19 & $\mathbf{v}$ \\
\hline Enter your Gender: & Male & (-) Female \\
\hline On Thyroxine : & Yes & No \\
\hline Query On Thyroxine: & Yes & (-) No \\
\hline On Anti-Thyroid Medication : & Yes & - No \\
\hline Sick: & Yes & (-) No \\
\hline Thyroid Surgery: & Yes & - No \\
\hline I131 Treatment: & Yes & (- No \\
\hline Query Hypothyroid : & Yes & - No \\
\hline Query Hyperthyroid : & Yes & - No \\
\hline Lithium : & Yes & (- No \\
\hline Goitre : & Yes & - No \\
\hline Tumor: & (-) Yes & No \\
\hline Hypopituitary : & Yes & (-) No \\
\hline Psych: & Yes & - No \\
\hline TSH Measured : & - Yes & No \\
\hline TSH : & \multicolumn{2}{|l|}{0.45} \\
\hline T3 Measured: & - Yes & No \\
\hline T3: & \multicolumn{2}{|l|}{3.2} \\
\hline 1T4 Measured : & - Yes & No \\
\hline Tा4 : & \multicolumn{2}{|l|}{130} \\
\hline T4U Measured : & (1) Yes & No \\
\hline T4U: & \multicolumn{2}{|l|}{1.83} \\
\hline FII Measured: & (-) Yes & No \\
\hline FTI : & \multicolumn{2}{|l|}{71} \\
\hline \multirow[t]{2}{*}{ TBG Measured: } & \multicolumn{2}{|l|}{ Yes } \\
\hline & \multicolumn{2}{|c|}{ Submit } \\
\hline
\end{tabular}

Fig 3: Input Screen to Identify Goiter

Output:

Report Generated as: Goiter.|3523

Referral Source Specified: STMW

Figure 3: This screen shot shows the diagnosis of Goiter according to the symptoms selected by the user, and as the range of the test results fed, the prediction is made and the refferal source STMW is suggested to the user for further proceedings of treatment. 


\subsection{Case-3:}

\begin{tabular}{|c|c|c|}
\hline \multicolumn{3}{|c|}{ SELECT THE SYMPTOMS } \\
\hline Enter your age : & 32 & $\mathbf{v}$ \\
\hline Enter your Gender : & Male & (1) Female \\
\hline On Thyroxine: & 0 Yes & (1) No \\
\hline Query On Thyroxine : & Yes & ○ No \\
\hline On Anti-Thyroid Medication : & $O$ Yes & No \\
\hline Sick: & 0 Yes & ( No \\
\hline Thyroid Surgery: & Yes & ( No \\
\hline I131 Treatment : & 0 Yes & (- No \\
\hline Query Hypothyroid : & Yes & ( ) No \\
\hline Query Hyperthyroid : & 0 Yes & ( ) No \\
\hline Lithium : & 0 Yes & ( ) No \\
\hline Goitre : & Yes & ( ) No \\
\hline Tumor: & (-) Yes & No \\
\hline Hypopituitary: & 0 Yes & No No \\
\hline Psych : & 0 Yes & (C) No \\
\hline TSH Measured : & (1) Yes & No \\
\hline TSH : & \multicolumn{2}{|l|}{1.7} \\
\hline T3 Measured: & () Yes & No \\
\hline T3: & \multicolumn{2}{|l|}{3.4} \\
\hline T14 Measured : & () Yes & No \\
\hline Tा4: & \multicolumn{2}{|l|}{120} \\
\hline T4U Measured: & - $)$ Yes & No \\
\hline T4U : & \multicolumn{2}{|l|}{1.5} \\
\hline FTI Measured: & - Y Yes & No \\
\hline FTI : & \multicolumn{2}{|l|}{80} \\
\hline \multirow[t]{2}{*}{ TBG Measured : } & \multicolumn{2}{|c|}{0 Yes } \\
\hline & \multicolumn{2}{|c|}{ Submit } \\
\hline
\end{tabular}

Fig 4: Input Screen for Insufficient Knowledge .

\section{Output:}

Report Generated as : Insufficient Data in the Knowledge Base

\section{\#32 F FFFFFTFFFFFFFT1.7T3.4T120T1.5T80F?}

Figure 4: Insufficient Knowledge in the Data Base: As the values fed by the user doesn't match with the data set or the Data Base, the Expert System simply displays a message saying that "Insufficient knowledge" and returns to the homepage.

\section{CONCLUSION}

Hypothyroid is one of the most common diseases. In its mildest form, hyperthyroid may not cause recognizable symptoms. More often, however, the symptoms are discomforting, disabling or even life-threatening. So, this HDES system aims to provide an imprecise diagnosis of the four thyroid related diseases such as Hyperthyroid, in particular Hypothyroid, goiter and T3 Toxic by accepting the values fed by the user and The EAS system is developed by using DMS which is applied on TDS to identify the relevant disease according to the data of the symptoms specified in the knowledge base. Once the user enters the details of the symptoms and submits then it predicts the Human disease which the user is suffering from and gives the referral source as the nearest hospital for further proceedings.

\section{APPENDIX}

The project "Health Diagnosis Expert Advisory System on Trained Data Sets for Hyperthyroid." is Java enabled web application developed using Java Server Pages and My SQL database. The following are the instructions that are to be followed in order to deploy the software.

The following are the requirements for successful deployment.

- Java enabled Web Server

- My SQL database, Datasets

- Linux/ Windows 98 or above

- Any java enabled web browser

\section{FUTURE ENHANCEMENTS}

The system is to be further enhanced using suitable Artificial Intelligence (AI), Optimization Algorithms or Machine Learning Algorithms in order to diagnose the disease and to provide a referral source as a nearest hospital. Here, we obtain the diagnostic methods for different symptoms entered by the user dynamically. If the data entered by the user is sufficient and, if it matches the Knowledge Base (KB) then it displays the actual disease caused by the thyroid gland, or else it displays the dialogue box stating that the knowledge is insufficient.

\section{REFERENCES}

[1] TDD via Hybrid Architecture Composing Rough Data Sets \& ML Algorithms., Second International Conference on Emerging Research in Computing, Information , Communication and Applications (ERCICA-2014) ., Preceding Published in ELSEVIER Volume 1, Issue 1, Pages: 307-316

[2] Plug In Generator To Produce Various Output For Unique Data., International Journal of Research in Engineering and Sciences( IJRES), Volume 2, Issue 2, Pages : $14-20$

[3] Offline Analysis \& Optimistic Approach on Livestock Expert Advisory System, Artificial Intelligent Systems and Machine Learning CIIT Journals , , Volume 5, Issue 12, Pages :488

[4] https://archive.ics.uci.edu/ml/datasets/Thyroid+Disease

[5] USING ARTIFICIAL NEURAL NETWORK IN DIAGNOSIS OF THYROID DISEASE: A CASE STUDY ,International Journal on Computational Sciences \& Applications (IJCSA) Vol.3, No.4, August 2013

[6] Expert System Based on Neural-Fuzzy Rules for Thyroid Diseases Diagnosis $@$ Springer-Verlag Berlin Heidelberg 2012

[7] Improve Computer-Aided Diagnosis with Machine Learning Techniques Using Undiagnosed Samples Ming $\mathrm{Li}$ and Zhi-Hua Zhou, Senior Member, IEEE ,2006

[8] Roman, W. Swiniarski, Andrzej Skowron," Rough Set Methods in Feature Selection and Recognition", Pattern Recognition Letters, vol. 24, pp. 833-849, 2003.

[9] Georg Peters, Richard Weber, Rene Nowatzke, "Dynamic Rough Clustering and its Applications", Applied Soft Computing, vol. 12, pp. 3193-3207, 2012. 
[10] Pawlak, Z., " Rough Sets- Theoretical Aspects of Reasoning about Data", Kluwer Academic, Dordrecht, 1991.

[11] Polkowski, L., Skowron, A.(Eds), Rough Sets in Knowledge Discovery, Physica- Verlag, Heidelberg, vols: 1 and 2, 1998

[12] Sara El-Sayed El-Metwally, Elsayed Radwan, Taher Hamza, " Multiple DNA Sequence Alignment using a Hybrid Model of GA and Rough Sets", Egyptian Computer Science Journal, vol. 34 no. 3, May 2010.

[13] CROCHEMORE, CZUMAJ -Speeding up two stringmatching algorithms -, et al. - 1994

[14] Thyroid Disease Diagnoses using C4.5 Algorithms and Data Mining Techniques ,2011

[15] Keles, A., "ESTDD: Expert System For Thyroid Diseases Diagnosis", Expert Syst. Appl. vol.34 no. 1,pp. 242-246, 2008

[16] Defend Data using ELGAMAL Digital Signature Data Decryption Algorithm., (IJCSIT) International Journal of Computer Science and Information Technologies, Volume 5 , Issue 4, Pages : 5062-5067

[17] Human Motion Detection Using Passive Infra Red Sensor., International Journal of Research in Computer Applications \& Information (C IASTER 2014, Volume 2, Issue 2, Pages :28-32.

\section{AUTHOR'S PROFILE}

Mr V Prasad, working as an Associate Professor in Raghu Institute of Technology since 8 Years having enough knowledge in Algorithms related to Machine Learning, Expert Systems \& Artificial Intelligence. He is awarded Bachelors Degree (Computers) from JNTU-H, Masters Degree (CST - AI \& Robotics) from Andhra University and currently pursuing $\mathrm{PhD}$ (Computers) in Gitam University. He had several publications in National / International Journals, and attended many National Seminars and presented papers in International conferences.

Dr T Srinivasa Rao, working as an Associate Professor in GITAM Institute of Technology ,GITAM UNIVERSITY since 10 Years and fetching a total experience of 13 Years . $\mathrm{He}$ had good expert knowledge in Software Engineering Advanced Computer Networks, Micro Processors, Embedded Systems, Databases and had rich journals published by him. He had guided many UG and PG Projects which are used in college / small firm levels also.He is now Placement Coordinator and Fellow and member of Professional bodies . A Good and Kind Hearted person

B Chaitanya, Final Year B.Tech in Raghu Institute of Technology affiliated to Andhra University, I am very interested in doing projects which are helpful for mankind. So I choosed Artificial Intelligence area to build this project by using my knowledge.

A Veera Reddy, Final Year B.Tech in Raghu Institute of Technology affiliated to Andhra University, Inspiring from the Artificial Intelligence, Expert system techniques. I tried to implement medical diagnosis expert system by using my knowledge which can be used by any unsophisticated user. 